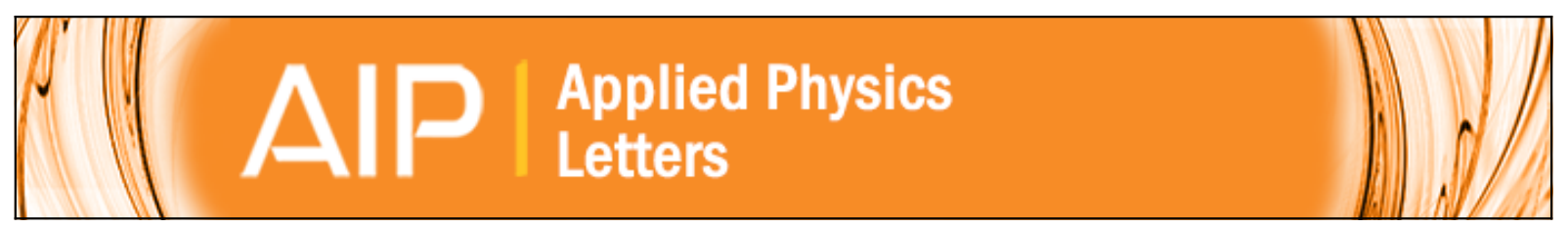

\title{
Structure-property relations in amorphous carbon for photovoltaics
}

Francesca Risplendi, Marco Bernardi, Giancarlo Cicero, and Jeffrey C. Grossman

Citation: Applied Physics Letters 105, 043903 (2014); doi: 10.1063/1.4891498

View online: http://dx.doi.org/10.1063/1.4891498

View Table of Contents: http://scitation.aip.org/content/aip/journal/apl/105/4?ver=pdfcov

Published by the AIP Publishing

\section{Articles you may be interested in}

Progressive structural and electronic properties of nano-structured carbon atomic chains

J. Appl. Phys. 113, 193704 (2013); 10.1063/1.4805047

Nanostructural interpretation for elastic softening of amorphous carbon induced by the incorporation of silicon and hydrogen atoms

J. Appl. Phys. 107, 124315 (2010); 10.1063/1.3431345

Density functional calculations on atomic and electronic structures of amorphous HfO 2 / Si ( 001 ) interface Appl. Phys. Lett. 95, 102905 (2009); 10.1063/1.3226636

Comparative analysis of electronic structure and optical properties of crystalline and amorphous silicon nitrides J. Appl. Phys. 106, 053717 (2009); 10.1063/1.3213359

Electron cyclotron resonance deposition, structure, and properties of oxygen incorporated hydrogenated diamondlike amorphous carbon films

J. Appl. Phys. 96, 5456 (2004); 10.1063/1.1804624

\section{AIP $\left.\right|_{\text {APL Photonics }}$}

APL Photonics is pleased to announce Benjamin Eggleton as its Editor-in-Chief

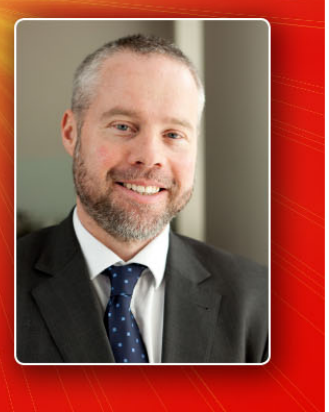




\title{
Structure-property relations in amorphous carbon for photovoltaics
}

\author{
Francesca Risplendi, ${ }^{1}$ Marco Bernardi, ${ }^{2}$ Giancarlo Cicero, ${ }^{1}$ and Jeffrey C. Grossman ${ }^{3, a)}$ \\ ${ }^{1}$ Dipartimento di Scienza Applicata e Tecnologia, Politecnico di Torino, 10129 Torino, Italy \\ ${ }^{2}$ Department of Physics, University of California, Berkeley, California 94720, USA \\ ${ }^{3}$ Department of Materials Science and Engineering, Massachusetts Institute of Technology, \\ Cambridge, Massachusetts 02139, USA
}

(Received 5 May 2014; accepted 16 July 2014; published online 28 July 2014)

Carbon is emerging as a material with great potential for photovoltaics (PV). However, the amorphous form (a-C) has not been studied in detail as a PV material, even though it holds similarities with amorphous Silicon (a-Si) that is widely employed in efficient solar cells. In this work, we correlate the structure, bonding, stoichiometry, and hydrogen content of a-C with properties linked to PV performance such as the electronic structure and optical absorption. We employ first-principles molecular dynamics and density functional theory calculations to generate and analyze a set of a-C structures with a range of densities and hydrogen concentrations. We demonstrate that optical and electronic properties of interest in PV can be widely tuned by varying the density and hydrogen content. For example, sunlight absorption in a-C films can significantly exceed that of a same thickness of a-Si for a range of densities and $\mathrm{H}$ contents in a-C. Our results highlight promising features of a-C as the active layer material of thin-film solar cells. (C) 2014 AIP Publishing LLC. [http://dx.doi.org/10.1063/1.4891498]

Carbon based solar cells have attracted renewed attention as a promising thin-film photovoltaic (PV) technology. ${ }^{1-4}$ Carbon nanomaterials, such as nanotubes, graphene, fullerenes, and their chemical derivatives, possess a range of favorable properties for PV. For example, thin films of carbon nanomaterials manufactured from solution can absorb a significant fraction of incident sunlight, while possessing chemical and thermal stability. ${ }^{2,5}$ Despite these advantages, carbon nanomaterials require precisely controlled structures to be employed in solar cells. For example, recent work has shown that carbon nanotubes with specific diameters and chiralities are necessary to optimize PV devices based on nanotubes and fullerenes; ${ }^{2,3}$ such stringent requirements pose at present significant challenges to fabricate large area carbon based solar cells. ${ }^{3}$ This scenario motivates a search for bulk carbon materials possessing the same desirable features of carbon nanomaterials coupled to large-scale manufacturability and low cost.

Amorphous carbon (a-C) holds a particularly promising role in carbon based PV and can be routinely deposited in thin-film form with accurate control of its structural and chemical properties. ${ }^{6}$ It is interesting to compare a-C with amorphous $\mathrm{Si}(\mathrm{a}-\mathrm{Si}) .{ }^{6}$ Due to its strong optical absorption and thin film manufacturability, hydrogenated a-Si (a-Si:H) has been investigated extensively for PV applications, resulting in solar cells with up to $\sim 10 \%$ efficiency. ${ }^{1}$ By contrast, application of a-C in solar cells has been very limited thus far. $^{7-10}$ While a-Si is commonly employed in its hydrogenated form a-Si:H to passivate dangling bonds, binding in a-C leads to $\mathrm{C}$ atoms in $s p, s p^{2}$, and $s p^{3}$ configurations, thus partially passivating dangling bonds in the absence of hydrogen. In addition, thin films of a-Si:H are subject to severe photodegradation processes, such as the Staebler-Wronski (SW) effect, which reduce the efficiency of a-Si:H solar

a) jcg@mit.edu cells. ${ }^{6,11,12}$ Interestingly, a-C does not possess an equivalent of the SW mechanism, likely due to the greater number of bonding possibilities of $\mathrm{C}$ atoms.

Theoretical modeling using first-principles calculations has contributed significantly to understand the structure of a$\mathrm{C},{ }^{13,14}$ and recent calculations have highlighted the potential of diamond-like a-C for sunlight harvesting. ${ }^{15}$ However, a systematic study of a-C and hydrogenated a-C $(\mathrm{a}-\mathrm{C}: \mathrm{H})$ aimed at exploring their applicability in solar cells has not been carried out.

In this work, we study the performance of a-C and a$\mathrm{C}: \mathrm{H}$ as materials for PV. We employ first-principles molecular dynamics (MD) simulations to generate sets of a-C and a$\mathrm{C}: \mathrm{H}$ structures with a range of densities and hydrogen contents. Using density functional theory (DFT) calculations, we correlate structural features, such as the density, $\mathrm{H}$ concentration, and fraction of $s p^{2}$ and $s p^{3}$ hybridized $\mathrm{C}$ atoms, with properties of relevance in PV such as the electronic structure, carrier mobility, and sunlight absorption. Our work suggests strategies to optimize these properties in a-C and highlights the promising features of a-C for application in solar cells.

We generated a-C and a-C:H structures using firstprinciples MD calculations based on DFT as implemented in the Vienna Ab-initio Simulation Package (VASP). ${ }^{16}$ Briefly, the Kohn-Sham (KS) Hamiltonian of the system included projector augmented wave pseudopotentials to describe the core-valence electron interaction ${ }^{17,18}$ and, in MD simulations, the local density approximation (LDA) of the exchange-correlation potential. ${ }^{19}$ In the MD simulations, the KS wavefunctions were expanded in plane waves with a kinetic energy cutoff of $350 \mathrm{eV}$, the Brillouin zone was sampled at the $\Gamma$ point, and the canonical (NVT) ensemble was adopted. Electronic and optical properties of the final structures were calculated by employing the HSE06 hybrid exchange functional. Optical absorption spectra were 
computed within the independent-particle approximation using the KS eigenvalues and wavefunctions, and including up to 1000 empty bands. Convergence with respect to all computed properties was carefully verified.

Our a-C systems consisted of cubic simulation cells with $216 \mathrm{C}$ atoms and densities in the $2.0-3.5 \mathrm{~g} / \mathrm{cm}^{3}$ range with increments in steps of $0.25 \mathrm{~g} / \mathrm{cm}^{3}$ for a total of seven different densities. In a-C:H, we introduced a variable fraction of $0 \%-20 \% \mathrm{H}$ atoms with increments of $10 \%$, for a total of three $\mathrm{H}$ concentrations. To obtain meaningful statistical averages of the quantities of interest, we prepared 10 samples for each density and $\mathrm{H}$ content.

The amorphous structures were prepared using a multistep protocol: the atomic positions were initially randomized using the algorithm from Wooten et al..$^{20}$ once randomized, the a-C and a-C:H samples were annealed at $5000 \mathrm{~K}$ for $3 \mathrm{ps}$ using first-principles MD as described above, and subsequently cooled to $300 \mathrm{~K}$ over $0.3 \mathrm{ps}$ and then further equilibrated for 3 ps. ${ }^{21}$ The structural analysis, including the radial distribution function, carbon coordination number and $s p^{3} / s p^{2}$ ratio, is discussed in the Supporting Information. ${ }^{22}$ Figure 1 summarizes the structural properties of our a-C and a-C:H structures with $\mathrm{H}$ content $0-20$ at. $\%$ and density values $\rho$ ranging between the density of graphite $(\rho \approx 2.0 \mathrm{~g} /$ $\left.\mathrm{cm}^{3}\right)$ and the density of diamond $\left(\rho \approx 3.5 \mathrm{~g} / \mathrm{cm}^{3}\right)$. Based on the number of first nearest neighbors of each $\mathrm{C}$ atom, reported in Figure 1(a) for three example a-C structures with different densities, we describe the $\mathrm{C}$ atoms as four-fold, three-fold, or two-fold coordinated (respectively, $\mathrm{C}_{4 c}, \mathrm{C}_{3 c}$, and $\mathrm{C}_{2 c}$ ). While $\mathrm{C}_{4 c}$ atoms correspond to $s p^{3}$ hybridization, $\mathrm{C}_{3 c}$ atoms can correspond to either $s p^{2}$ hybridized $\mathrm{C}$ atoms or $s p^{3}$ hybridized $\mathrm{C}$ atoms with a dangling bond. $\mathrm{C}_{2 c}$ corresponds to $s p$ hybridization and was less commonly observed in our samples. Our analysis indicates that the fraction of $\mathrm{C}_{4 c}, \mathrm{C}_{3 c}$, and $\mathrm{C}_{2 c}$ atoms depends strongly on the density and
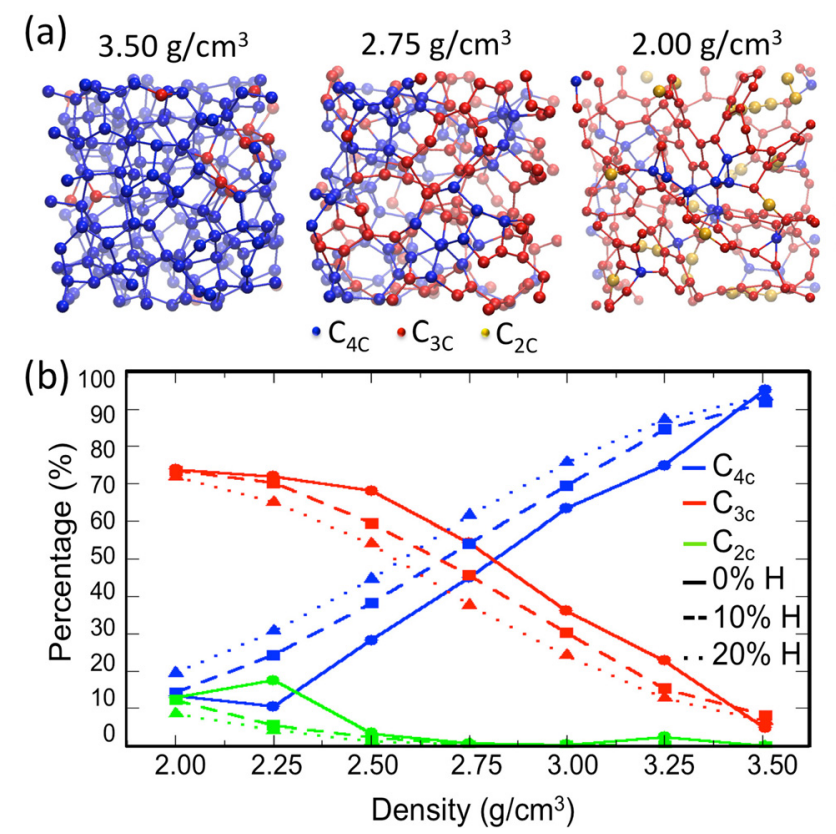

FIG. 1. (a) Representative structures and bonding in a-C samples with different densities $2.00,2.75$, and $3.50 \mathrm{~g} / \mathrm{cm}^{3}$. Blue, red, and yellow spheres represent, respectively, $\mathrm{C}_{4 c}, \mathrm{C}_{3 c}$, and $\mathrm{C}_{2 c}$ atoms. (b) Average fraction of $\mathrm{C}_{4 c}$, $\mathrm{C}_{3 c}$, and $\mathrm{C}_{2 c}$ atoms as a function of density and $\mathrm{H}$ content.
$\mathrm{H}$ content and their average trends are reported in Figure 1(b).

Next, we explore the electronic and optical properties. Figure 2(a) shows the electronic density of states (DOS) of $\mathrm{a}-\mathrm{C}$ and $\mathrm{a}-\mathrm{C}: \mathrm{H}$ structures as a function of the density and $\mathrm{H}$ content. For a-C with $\rho=3.5 \mathrm{~g} / \mathrm{cm}^{3}$, we observe the presence of a band gap of $\sim 5 \mathrm{eV}$, close to the value of the DFT (HSE06) gap of diamond. As $\rho$ decreases towards the density of graphite, we observe a decrease in the band gap due to the appearance of states with energy within the band gap, belonging to the so-called Urbach tails of the DOS. ${ }^{6}$ At a density of $\rho \approx 3 \mathrm{~g} / \mathrm{cm}^{3}$, the gap closes completely, and for lower densities, the Urbach tails extend further into the gap (see Figure S2 of Supporting Information). Analysis of the projected DOS of C atoms (not shown here) reveals that the top of the valence band and the bottom of the conduction band at densities of $3-3.5 \mathrm{~g} / \mathrm{cm}^{3}$ are contributed mostly by $p_{z}$ states of $s p^{2} \mathrm{C}$ atoms, while $s$ and $p$ states of $s p^{3} \mathrm{C}$ atoms correspond to energies deeper inside the valence and conduction bands. At densities lower than $3 \mathrm{~g} / \mathrm{cm}^{3}$, we observe the formation of extended $\pi$ states contributed by $p_{z}$ states of threefold coordinated $\mathrm{C}$ atoms and leading to the Urbach tail formation. This observation is consistent with the closure of the gap as the $s p^{2}$ fraction increases in structures with lower densities. We conclude that the value of the band gap is determined by the conjugation length of $\pi$ states from $s p^{2} \mathrm{C}$ atoms within the a-C network, similar to trends observed in conjugated molecules. ${ }^{23-25}$ Our results show that lower densities in a-C lead to larger fractions of $s p^{2} \mathrm{C}$ atoms, and thus to more extensive $\pi$ conjugation and reduced band gaps. We observed similar trends in a-C:H, with the important difference that the disorder induced by the $\mathrm{H}$ atoms further increases the number of states in the Urbach tails (Figure 2(a)).

Figure 2(b) shows the charge density associated with KS states of different energies in a-C with $\rho=3.5 \mathrm{~g} / \mathrm{cm}^{3}$. We observe that orbital localization depends strongly on the energy of KS states near the Fermi energy $\left(E_{F}\right)$. Localized

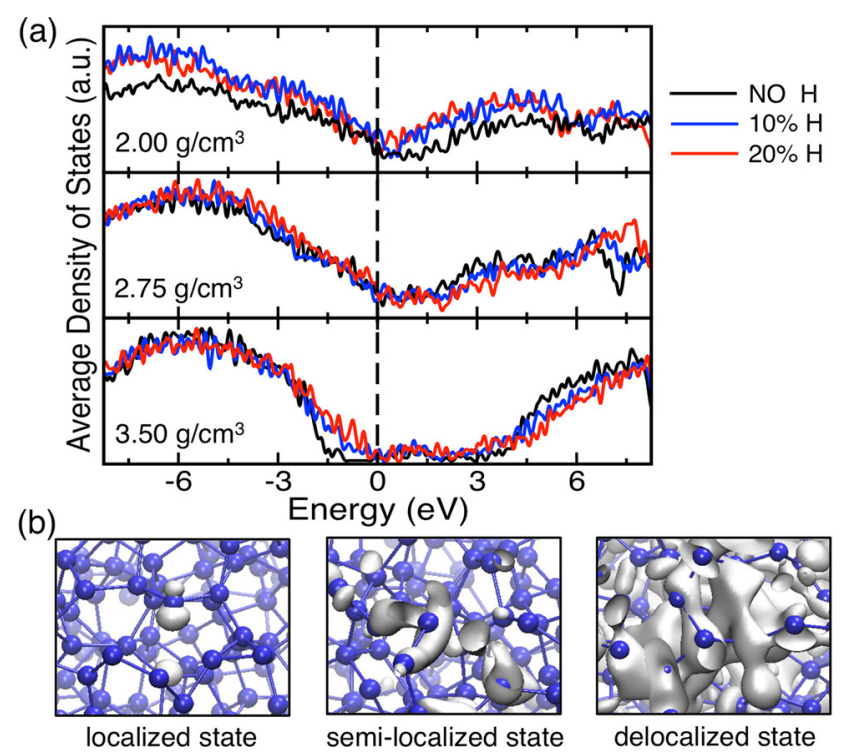

FIG. 2. (a) DOS of a-C samples as a function of density and $\mathrm{H}$ concentration. (b) Charge density (shown in gray) associated with a localized orbital (left panel), a semilocalized orbital (middle panel), and a delocalized orbital (right panel) in a-C for a sample with $\rho=3.5 \mathrm{~g} / \mathrm{cm}^{3}$. 
states found at energies close to $\mathrm{E}_{F}$ arise from dangling bonds, are typically localized on a few $\mathrm{C}$ atom sites and associated with the presence of dangling bonds in the a-C network. Progressively more delocalized states are found at lower (higher) energies within the valence (conduction) band. Such states of intermediate delocalization are found to be associated with weak disorder such as bond angle distortion. Finally, states delocalized over the entire simulation cell are found at higher energies within the valence and conduction bands.

In amorphous semiconductors, it is important to locate the energies in the valence and conduction bands marking a transition between localized states contributing negligibly to charge transport and delocalized states contributing to charge transport. These energies are called valence/conduction band mobility edges-respectively, $E_{m}^{V}$ and $E_{m}^{C}$ - and their difference is the mobility gap $E_{G}=E_{m}^{C}-E_{m}^{V}$. For solar energy conversion, mobility gaps smaller than $2-3 \mathrm{eV}$ are desirable to ensure that photogenerated carriers possess sufficiently large diffusion lengths to be collected at the electrodes. To compute the mobility edges in a-C and a-C:H, we quantified the localization of the KS orbitals by evaluating their inverse participation ratios (IPR) following the procedure in Ref. 26. We observed sharp transitions in the IPR values for increasing energies both in the valence and conduction bands, thus allowing us to identify the mobility edges as the energies of the IPR value transition, and consequently compute the mobility gaps.

Figure 3 shows the mobility gap as a function of density and $\mathrm{H}$ content. For a-C, we observe $E_{G}$ values in the $0-6 \mathrm{eV}$ range increasing monotonically with density. The increase of $E_{G}$ as a function of density is associated with a decreasing overlap of $\pi$ states from three-fold coordinated $\mathrm{C}$ atoms. The introduction of $10 \%-20 \% \mathrm{H}$ atoms shifts up the $E_{G}$ range to $1.5-6.5 \mathrm{eV}$ but does not alter the trends. Overall, we observe that mobility gaps of less than $\sim 3 \mathrm{eV}$ as desirable in PV can be achieved at small to intermediate densities of up to $2.5 \mathrm{~g} / \mathrm{cm}^{3}$ in a-C and $2.25 \mathrm{~g} / \mathrm{cm}^{3}$ in a-C:H. We found that the same density ranges are also ideal for sunlight absorption, as discussed next.

The discussion of structural and electronic properties in a$\mathrm{C}$ and a-C:H carried out thus far forms the basis for our study of sunlight absorption in a-C. Figure 4(a) shows the calculated optical absorption coefficient $\alpha$ (units of $\mathrm{cm}^{-1}$ ) of nonhydrogenated a-C for samples of different densities. We further computed the Tauc optical gaps $E_{t}^{29}$ by applying a linear fit of $(\alpha E)^{1 / 2} v s E$, where $E$ is the photon energy (see Figure S5 of Supporting Information).

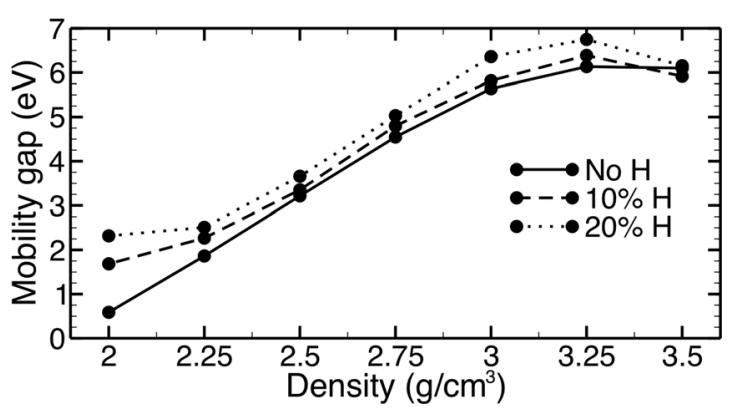

FIG. 3. Mobility gaps as a function of density and $\mathrm{H}$ concentration.
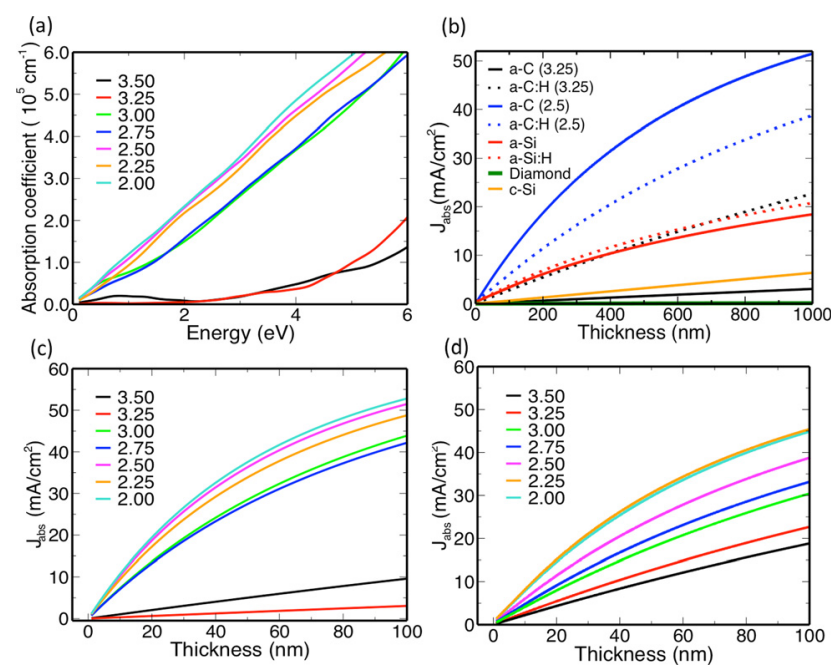

FIG. 4. (a) Absorption coefficient in the solar photon energy range, shown as a function of density for non-hydrogenated a-C. (b) Comparison of photogenerated current density in amorphous and crystalline structures based on $\mathrm{C}$ and $\mathrm{Si}$, for film thickness values of $0-100 \mathrm{~nm}$. The silicon density in a-Si and a-Si:H is equal to $2.37 \mathrm{~g} / \mathrm{cm}^{3}$, and the $\mathrm{H}$ content corresponds to $20 \%{ }^{30}$ (c) Photogenerated current in a-C as a function of thickness for structures of different densities in the $2.00-3.50 \mathrm{~g} / \mathrm{cm}^{3}$ range. (d) Photogenerated current in a-C:H with $20 \% \mathrm{H}$, shown here as a function of thickness for structures of different densities in the $2.00-3.50 \mathrm{~g} / \mathrm{cm}^{3}$ range.

At all density values, we observe relatively large $\alpha$ values of approximately $10^{5} \mathrm{~cm}^{-1}$ at visible photon energies, comparable to absorption values in a-Si (calculated at the same level of theory) and in the best organic and inorganic absorbers employed in $\mathrm{PV} .{ }^{27}$ The large absorption values in the visible make a-C (and a-C:H) an excellent candidate material for sunlight harvesting in PV. We observe that absorption at visible energy is mainly determined by inter-band transitions between $\pi$ and $\pi^{*}$ states from $s p^{2} \mathrm{C}$ atoms, consistent with our electronic structure results. For low density structures with a large fraction of $s p^{2} \mathrm{C}$ atoms, Figure 4(a) shows large absorption values due to the presence of delocalized $\pi$ and $\pi^{*}$ states with extended conjugation. By contrast, samples with densities higher than $3 \mathrm{~g} / \mathrm{cm}^{3}$ and lower $\pi$ conjugation show absorption onsets at higher energies and can absorb sunlight significantly only at energies larger than $2.5-3 \mathrm{eV}$. These trends suggest that low density a-C structures favoring extended $\pi$ conjugation are preferable for sunlight collection and use in PV. Optical absorption in a-C:H shows a similar behavior, with subtle differences due to structural effects imparted by $\mathrm{H}$ atoms (see Figures S3 and $\mathrm{S} 4$ of Supporting Information). In particular, $\mathrm{H}$ atoms enhance absorption in samples with higher densities as they introduce defect states close to the Fermi energy, and reduce absorption in samples with lower densities by saturating $\mathrm{C}=\mathrm{C}$ double bonds. The ability to tune sunlight absorption by changing the density and $\mathrm{H}$ content (and as a result the fraction of $s p^{2}$ atoms and the conjugation) is unique to a-C and a-C:H. Indeed $\mathrm{Si}$ atoms do not form bulk compound with $s p^{2}$ hybridization and only few stable molecules are known to present $\mathrm{Si}=\mathrm{Si}$ double bonds (see for example Ref. 28).

To further quantify sunlight absorption in a-C and a$\mathrm{C}: \mathrm{H}$, we combined the computed absorption coefficients with the incident AM1.5 solar spectrum (see Supporting Information) to calculate the generated photocurrent density 
$J_{a b s}$ (units of $\mathrm{mA} / \mathrm{cm}^{2}$ ) under AM1.5 illumination. We computed $J_{a b s}$ as a function of thickness for a-C and a-C:H films using the formula

$$
J_{a b s}=\int_{0}^{\infty} d E J_{p h}(E)\left[1-e^{-\alpha(E) L}\right],
$$

where $J_{p h}(E)$ is the incident AM1.5 solar flux at photon energy $E$, and $L$ is the thickness. We remark that $J_{a b s}$ is an upper estimate for the short-circuit current generated in a slab of a material of thickness $L$ and absorption coefficient $\alpha(E)$. Figure 4(b) shows the generated photocurrent in a-C and a-C:H samples with two different densities $\left(2.5 \mathrm{~g} / \mathrm{cm}^{3}\right.$ and $3.25 \mathrm{~g} / \mathrm{cm}^{3}$, respectively), compared to the same quantity for a-Si and a-Si:H calculated using the same approach. ${ }^{30}$ Crystalline $\mathrm{Si}$ and diamond are also compared for reference. We observe that the photocurrent $J_{a b s}$ increases significantly for a decrease in density from $3.25 \mathrm{~g} / \mathrm{cm}^{3}$ to $2.5 \mathrm{~g} / \mathrm{cm}^{3}$. Hydrogenation has only a minor effect in the low density sample, but significantly enhances sunlight absorption in the high density sample. On the other hand, hydrogenation has a minor effect on the absorption of a-Si (see Figure 4(b)).

Our results demonstrate that $\mathrm{a}-\mathrm{C}$ can provide absorption much superior to crystalline $\mathrm{Si}$ and diamond (the gap of which is too large to absorb sunlight appreciably). Moreover, by properly tuning the density and the hydrogen content (and thus the $\pi$ conjugation), the absorbed sunlight and the resulting photocurrent can be made larger than a-Si, by as much as a factor of 2 for the same thickness. By contrast, a-Si and a$\mathrm{Si}: \mathrm{H}$ tend to form large fractions of $s p^{3}$ atoms due to the different hybridization of the $p$ states in Si. For this reason, absorption in a-Si and a-Si:H samples is mostly determined by transitions between electronic states in distorted $s p^{3} \mathrm{Si}-\mathrm{Si}$ bonds contributing to the Urbach tails.

Taken together, our results show that sunlight absorption in a-C is strong and can be tuned by orders of magnitude by tailoring the composition and deposition conditions. Experimentally, such density and hydrogenation control can be achieved routinely ${ }^{6}$ and could be applied to prepare high quality a-C and a-C:H solar cell active layers. While our results establish that a-C can achieve comparable sunlight absorption to a-Si, the successful preparation of a PV device based on a-C would require additional steps, including a suitable device architecture to extract electrons and holes at separate contacts, as well as the reduction of sources of carrier recombination to achieve large currents and voltages. Solar cell devices consisting of $\mathrm{a}-\mathrm{C}$ as the active layer material may provide a pathway to harvest and convert sunlight in large area carbon based solar cells.
The authors thank NERSC and XSEDE for providing computational resources. This work was partially supported by the Eni Solar Frontiers Program at MIT. F.R. and G.C. acknowledge the Politecnico di Torino-MIT MITOR project funded by the Fondazione Compagnia di San Paolo.

${ }^{1}$ D. Ginley, M. Green, and R. Collins, MRS Bull. 33, 355 (2008).

${ }^{2}$ M. Bernardi, J. Lohrman, P. Kumar, A. Kirkeminde, N. Ferralis, J. Grossman, and S. Ren, ACS Nano 6, 8896 (2012).

${ }^{3}$ R. Jain, R. Howden, K. Tvrdy, S. Shimizu, A. Hilmer, T. McNicholas, K. Gleason, and M. Strano, Adv. Mater. 24, 4436 (2012).

${ }^{4}$ M. P. Ramuz, M. Vosgueritchian, P. Wei, C. Wang, Y. Gao, Y. Wu, Y. Chen, and Z. Bao, ACS Nano 6, 10384 (2012).

${ }^{5}$ H. Zhu, J. Wei, K. Wang, and D. Wu, Sol. Energy Mater. Sol. Cells 93, 1461 (2009).

${ }^{6}$ R. A. Street, Technology and Applications of Amorphous Silicon (Springer, 2000).

${ }^{7}$ Z. Q. Ma and B. X. Liu, Sol. Energy Mater. Sol. Cells 69, 339 (2001).

${ }^{8}$ J. Han, M. Tan, J. Zhu, S. Meng, B. Wang, S. Mu, and D. Cao, Appl. Phys. Lett. 90, 083508 (2007).

${ }^{9}$ H. A. Yu, Y. Kaneko, S. Yoshimura, and S. Otani, Appl. Phys. Lett. 68, 547 (1996).

${ }^{10}$ Z. B. Zhoua, R. Q. Cuia, Q. J. Panga, G. M. Hadia, Z. M. Dingb, and W. Y. Lib, Sol. Energy Mater. Sol. Cells 70, 487 (2002).

${ }^{11}$ D. L. Staebler and C. R. Wronski, J. Appl. Phys. 51, 3262 (1980).

${ }^{12}$ A. Shah, P. Torres, R. Tscharner, N. Wyrsch, and H. Keppner, Science 285, 692 (1999).

${ }^{13}$ G. Galli, R. Martin, R. Car, and M. Parrinello, Phys. Rev. Lett. 62, 555 (1989).

${ }^{14}$ N. A. Marks, D. R. McKenzie, B. A. Pailthorpe, M. Bernasconi, and M. Parrinello, Phys. Rev. Lett. 76, 768 (1996).

${ }^{15}$ G. Tritsaris, C. Mathioudakis, P. Kelires, and E. Kaxiras, J. Appl. Phys. 112, 103503 (2012).

${ }^{16}$ G. Kresse and J. Furthmüller, J. Phys. Rev. B 54, 11169 (1996).

${ }^{17}$ P. E. Blöchl, Phys. Rev. B 50, 17953 (1994).

${ }^{18}$ G. Kresse and D. Joubert, Phys. Rev. B 59, 1758 (1999).

${ }^{19}$ J. P. Perdew and A. Zunger, Phys. Rev. B 23, 5048 (1981).

${ }^{20}$ F. Wooten, K. Winer, and D. Weaire, Phys. Rev. Lett. 54, 1392 (1985).

${ }^{21}$ We have verified that our cooling rate was sufficiently slow by comparing the results with those obtained with simulations with $10 \mathrm{X}$ slower cooling rates. Before performing electronic structure calculations, all structures were relaxed within DFT to less than $10^{-4} \mathrm{eV} / \AA$ in the residual atomic forces.

${ }^{22}$ See supplementary material at http://dx.doi.org/10.1063/1.4891498 for a more detailed structural analysis.

${ }^{23}$ J. Rocali, Macromol. Rapid Commun. 28, 1761 (2007).

${ }^{24}$ J. Hou, M. Park, S. Zhang, Y. Yao, L. M. Chen, J. H. Li, and Y. Yang, Macromolecules 41, 6012 (2008).

${ }^{25}$ M. Zamadar, S. Asaoka, D. C. Grills, and J. R. Miller, Nat. Commun 4, 1 (2013).

${ }^{26}$ T. A. Abtew, M. Zhang, and D. A. Dra, Phys. Rev. B 76, 045212 (2007).

${ }^{27}$ L. Yu and A. Zunger, Phys. Rev. Lett. 108, 068701 (2012).

${ }^{28}$ D. F. Perepichka and F. Rosei, Small 2, 22 (2006)

${ }^{29}$ J. Tauc, R. Grigorovici, and A. Vancu, Phys. Status Solidi B 15, 627 (1966).

${ }^{30} \mathrm{We}$ used cubic simulation cells containing $216 \mathrm{Si}$ atoms for the a-Si samples and introduced $20 \% \mathrm{H}$ atoms in the case of a-Si:H. The amorphous structures were prepared using the same multi-step MD protocol employed for $\mathrm{a}-\mathrm{C}$ and $\mathrm{a}-\mathrm{C}: \mathrm{H}$. The optoelectronic properties of the final systems were averaged over ten samples. 